\title{
Research on Fashion Clothing Supply Chain Decision-Making Based on Strategic Consumption
}

\author{
Xing-jian Zhou ${ }^{1,2,}$, Yi-Ping $\mathrm{Xu}^{3}$, Wen-ke Jiang ${ }^{1}$ and Hao Zhang ${ }^{1}$ \\ ${ }^{1}$ School of Management, Wuhan Textile University Wuhan, China \\ ${ }^{2}$ Research Center of Enterprise Decision Supportion, Hubei Province Research base for Humanities and Social \\ Sciences Wuhan, China \\ ${ }^{3}$ Logistics Engineering College, Wuhan University of Technology Wuhan, China \\ Email: wuliuwtu@163.com
}

\begin{abstract}
The rational behavior of strategic consumers is introduced into the operation and management of fashion clothing supply chain to analyze the optimal pricing decision. Based on the inconsistent evaluation of fashion clothing products by consumers, and the influence of rational behavior of strategic consumers on the supply chain decision-making, the further expansion is made to discuss the retailers' decision-making under the quantities restriction commitment.It is found that when consumers have rational behavior of waiting, the optimal inventory of supply chain decreases under the premise of satisfying consumers'needs. In order to cope with this effect, customers have a more accurate judgment on the quantity of products available when sellers implement strategies such as limited issuance and collection edition. Therefore, it reduces the likelihood that customers will wait and see, and is more likely to buy in the peak season.
\end{abstract}

Keywords: Fashion clothing supply chain, strategic consumers, Newsboy model, rational expectation equilibrium

\section{基于策略型消费的时尚服装供应链决策研究}

\author{
周兴建 ${ }^{1,2}{ }^{*}$, 徐伊平 ${ }^{3}$, 姜文可 ${ }^{1}$, 张昊 ${ }^{1}$ \\ 1 武汉纺织大学 管理学院, 武汉 \\ 2 湖北省人文社会科学研究基地 企业决策支持研究中心, 武汉 \\ 3 武汉理工大学 物流工程学院, 武汉 \\ Email: wuliuwtu@163.com
}

\begin{abstract}
摘要: 将策略型消费者的理性行为引入到时尚服装供应链运营管理中, 分析其对时尚服装供应链运营 及定价决策的影响。基于消费者群体对时尚服装品的评价不一致，对销售商、终端消费者组成的供应 链分析策略型消费者的理性行为对消费者决策的影响，并在此基础上进一步做出扩展，讨论销售商采 用数量限制承诺下销售商决策。经分析发现，当消费者具有观望和等待的理性行为后，在满足消费者 需求的前提下，供应链的最优库存量有所降低; 为应对这种影响, 当销售商实施限量发行、珍藏版等 策略时, 客户对产品的可得数量有比较准确的判断, 因此降低了客户等待观望的可能性, 更倾向于在 旺季时购买。
\end{abstract}

关键词：时尚服装供应链；策略型消费者；报童模型；理性期望均衡

\section{1 引言}

时尚服装品具有时尚性、流行性、季节性、易贬值等特征，以消费者为主体的需求导向明显。而终端消 费者购买决策逐步具有策略性，对厂商未来打折及供货走势形成理性预期，由此来选择购买时机，从而 获取最大的消费者剩余。这种行为复杂性对供应链节点的定价、库存策略以及收益的影响已不可忽略, 
因此, 如何在决策模型中考虑消费者理性行为, 以及如何引入应对机制, 提高供应链收益, 已引起众多 学者的关注。如, 路应金等 ${ }^{[1]}$ (2017) 对基于顾客理性行为的数学建模进行综述分析; 曲佳莉等 ${ }^{[2]}$ (2017) 研究了基于策略型消费者的理性行为, 销售商对供货配给及售销价的最优决策, 分析了消费者购买及等 待的理性决策; 张学龙等 ${ }^{[3]}$ (2018) 利用消费者的学习过程, 建立了供应链成员的库存补充数学模型, 分 析在此条件下各方的最优决策; 李玮珩 ${ }^{[4]}$ (2017) 利用终端消费者的异构价值评估假设, 对供应链中广泛 使用的回购决策进行了分析, 得出了有别于需求外生给定模型的结论; 苪鸿峰[5]（2017）研究了存在顾 客策略行为情况下, 快速补货运营策略对商家收益的影响; 李艳冰等 ${ }^{[5]}$ (2018) 基于顾客策略行为讨论了 差价补偿、二部定价的协调效果。张正林 ${ }^{[6]}$ (2014) 从消费者的购买决策模型出发,提炼出网络定价的三 个特点:多变性、透明性和智能化,探讨了这三个特点在消费者五个不同决策阶段的作用,并有针对性地提 出电商网站相应的策略思路; 刘甜甜 ${ }^{[7]}$ (2018) 从消费者的决策心理为切入点, 阐述了消费者的决策心理 的重要性,进而提出电子智能产品的营销策略。陈静 ${ }^{[8]}$ （2017）基于以上背景,在考虑消费者策略行为的基 础上, 以销售利润最大化为目标, 研究了销售两种不同等级可相互替代产品的企业的最优跨期定价问题,并 与消费者短视型的情况进行了对比。

以往的文献很少引入终端消费者的理性行为对时尚服装供应链运作的影响, 主要是基于所有消费者对 产品估值一致的前提下展开, 而时尚服装供应链客户需求复杂, 使其实际运作面临诸多特点, 如因时尚 规律而需求变化特征突出, 根据时尚阶段不同, 消费者行为体现出品牌忠诚度、流行、跟风等阶段性需 求 ${ }^{9}$; 单次生产的供需不平衡及消费者观望行为而引发换季需求, 更加强调准确把握终端消费者的决策, 从而预测到市场需求的变化, 完成对库存、定价及销售的有效决策 ${ }^{[10-12]}$ 。本文将放松消费者对产品估值 一致的假设, 在消费者预评价基于一定随机分布的前提下展开讨论, 并将引入理性期望均衡(Rational Expectation Equilibrium, REE)的概念来分析合作各方的行为, 即所有参与者是理性的: 1)生产商将基 于对消费者消费行为的理性期望, 决定生产的产品数量和销售价格; 2)消费者将基于对产品的效用及可 得性的期望, 决定是否购买或推迟购买产品。

\section{2 问题描述}

假设某时尚服装推向市场时, 面临一个报童型的不确定客户群体 $D$, 设需求 $x$ 是连续的随机变量, 其概 率密度函数为 $f(x)$, 分布函数 $F(q)=\int_{0}^{q} f(x) d x$ 是连续可微的, 且具有递增的风险故障率 (Increasing failure rate, IFR), 即 $\frac{f(x)}{\bar{F}(x)}$ 递增, 其中 $\bar{F}(x)=1-F(x)$, 为销售商及消费者的共有信息。生产商以边际成本 $c$ 生产 $q$ 单位产品, 并在旺季以销售价格 $P_{1}$ 推向市场。旺季过后, 产品可能会有剩余, 销售商会以打折价 $P_{2}$ 进行清仓, 且 $P_{2}<P_{1}$ 。

在消费者群体 $x$ 中, 此服装对每一单个消费者产生的效用不同（对应每个消费者对同一服装产品的评 价不同）, 假设可能达到的最高效用为 $K_{i}$, 其中 $i=\{1,2\}$, 表示此时尚服装的旺季及换季时的 2 个销售 阶段; 由于消费者群体在旺季时对服装的总体评价比较高, 等到过季时会根据时尚的衰落而有所下降, 因此不失一般性, 假设 $V_{2}<V_{1}$ 。产品对每个消费者产生的效用为 $V_{i}$ 乘以消费者的类型 $\theta, \theta$ 为消费者的私 有信息，在 $[0,1]$ 上密度函数和分布函数分别为 $g(\theta) 、 G(\theta)$ 。

消费者的保留效用为 $m$ （即如果购买产品最后的净效用小于 $m$, 消费者将放弃购买）；消费者评估是 否以价格 $P_{1}$ 购买或推迟到换季时以打折价 $P_{2}$ 购买。此时未购买的消费者可以比全价 $P_{1}$ 更低的 $P_{2}$ 得到产 品, 获得更高的剩余效用, 同时承担产品在前一阶段全部销售完, 存在购买不到产品的风险。

\section{3 消费者决策分析}

\section{1 消费者的效用函数}

消费者将在 2 个阶段的效用间权衡应该在哪个阶段购买: 旺季购买时, 以较高的价格一定可以买到产品, 并提前享用; 打折期购买时, 可能以较低的价格购买, 但是有可能剩余产品有限, 出现断码等情形, 而 无法完成购买。本文将用打折期产品可得的概率 $\varphi$ 描述, 从而求得消费者在换季时的期望剩余效用。只有 在 2 个阶段的剩余相等时消费者在任何一个阶段购买都无差异, 即:

$$
\theta_{h} V_{1}-p_{1}=\left(\theta_{h} V_{2}-p_{2}\right) \hat{\varphi}
$$

式中 $\hat{\varphi}$ 为客户对打折产品可得率的期望, $\hat{x}$ 为理性期望。

命题 1: 存在一个 $\theta_{h}^{*}$, 当消费者对产品的评价 $\theta$ 满足 $\theta>\theta_{h}^{*}$ 时, 应该选择在旺季时购买。其中 
部分消费者将权衡是否购买产品：

$$
\theta_{h}^{*}=\frac{p_{1}-\hat{\varphi} p_{2}}{V_{1}-\hat{\varphi} V_{2}}
$$

$$
\left(\theta_{1} V_{2}-p_{2}\right) \hat{\varphi} \geq 0
$$

命题 2: 存在一个 $\theta_{1}^{*}$, 当消费者对产品的评价 $\theta$ 满足 $\theta_{h}^{*}>\theta>\theta_{1}^{*}$ 。十时, 应该选择在打折时购买。其中 $\theta_{1}^{*}=$ $\frac{p_{2}}{V_{1}}$, 且 $\theta_{1}^{*}<\theta_{h}^{*}$ ； 若消费者对产品的评价 $\theta<\theta_{1}^{*}$, 将不会购买该产品。

由 $\theta_{h}^{*}$ 及 $\theta_{1}^{*}$ 的表达式可知 $\theta_{1}^{*}$ 与旺季的价格 $P_{1}$ 无关, 只与消费者对打折后的产品总估价 $V_{2}$ 及打折价 $P_{1}$ 有关; 而当全价 $P_{1}$ 越高, 则 $\theta_{h}^{*}$ 也越高, 即愿意在旺季购买的消费者预评估下限也越高, 这与直觉相符。 另外 $\theta_{h}^{*}$ 也间接地通过 $\hat{\varphi}$ 销售商的库存量影响。

市场容量在不同销售季节之间的分配如下: 部分评价高的消费者 $\bar{G}\left(\theta_{h}^{*}\right) x$ 全价购买, 部分评价较低的 消费者 $\left[\bar{G}\left(\theta_{1}^{*}\right)-\bar{G}\left(\theta_{h}^{*}\right)\right] x$ 会等待打折购买, 另外还有一部分评价很低的消费者 $\bar{G}\left(\theta_{1}^{*}\right) x$ 无法承受销售价格, 因此放弃购买。

图 1 为消费者购买决策对序图。描述了根据客户对产品的评价不同，在不同销售季节的购买决策。

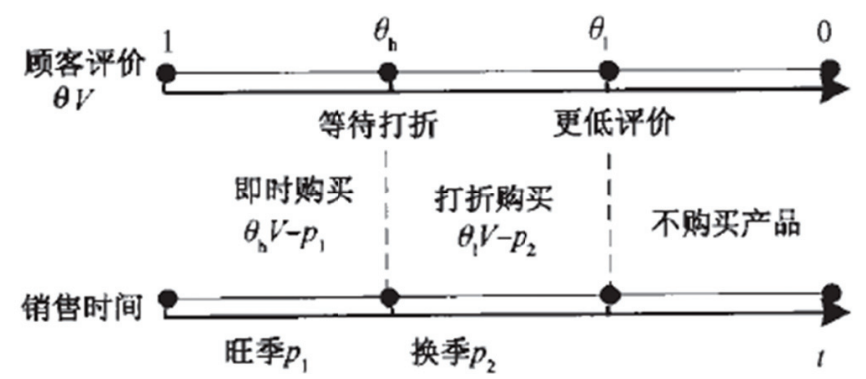

图 1. 消费者购买决策时序图

\section{2 理性期望均衡}

消费者在打折期是否能购得产品的可得率取决于销售商在旺季销售时的库存量 $q$ 。若 $q$ 单位产品在旺季 销售一空, 则消费者无法在换季时以打折价获得产品; 若 $q$ 单位产品的旺季销售有所剩余, 即 $\bar{G}\left(\theta_{h}^{*}\right) x<q$, 则消费者才有可能进行打折购买, 因此, 消费者将对销售商的产品量 $q$ 有一个预期, 并基于这种预期, 进行购买或等待的决策; 相应地, 销售商预见到消费者会有这种权衡行为, 考虑到这种因素, 会对消费 者所能承受的价格形成一个预期 $\hat{p}_{1}$, 完成定价, 并决定自己的库存量 $q$, 从而最大化其效益。

本文假设所有的参与者都是理性决策者, 延用文献的描述形式, 可知此博弯过程将达到理性均衡 $\left\{P_{1}, q\right.$, $\left.\hat{p}_{1}, \hat{\varphi}\right\}$, 基于期望 $\hat{p}_{1} 、 \hat{\varphi}$, 双方都会选择均衡结果, 并跟最后实现的决策相同。此时销售商将对消费者 将能承受的价格有一个正确的期望, 选定的均衡销售价 $\hat{p}_{1}$ 将等于客户的保留期望值 $P_{1}$, 达到客户在打折 时购买的相同效用, 即 $p_{1}=\theta_{h} V_{1}-\left(\theta_{h} V_{2}-p_{2}\right) \hat{\varphi}$; 另一方面, 客户对旺季销售所剩下产品数量的期望是符 合市场总体需求分布的, 即 $\hat{\varphi}=F\left(q^{*}\right)$, 其中 $q^{*}$ 为销售商最大化销售收益的库存量, $q^{*}=\arg _{q} \max \Pi\left(q, p_{1}\right)$ 。

\section{4 销售商决策分析}

\section{1 销售商的期望收益}

销售商的期望收益为:

$$
\prod\left(q, p_{1}\right)=\left(p_{1}-p_{2}\right) E\left(\bar{G}\left(\theta_{h}^{*}\right) x \wedge q\right)-\left(c-p_{2}\right) q
$$

为了对比起见, 本文假设经典报童模型下

$$
\prod_{0}=\left(p_{1}-p_{2}\right) E(x \wedge q)-\left(c-p_{2}\right) q
$$


可得

$$
q_{0}^{*}=\bar{F}^{-1}\left(\frac{c-p_{2}}{p_{1}-p_{2}}\right)
$$

结论 1: 当达到均衡时, 销售商采用以下的 $\left\{p_{1}^{*}, q^{*}\right\}$ 决策, 将最大化旺季销售收益 $q^{*}=\bar{G}\left(\theta_{h}^{*}\right) q_{0}^{*}$ 且 $\Pi\left(q^{*}, P_{1}\right)=$ $\bar{G}\left(\theta_{h}^{*}\right) \Pi_{0}$ 。为了简洁起见, 本文假设旺季及打折时, 消费者群体对产品的估值上限相同, 即 $V_{1}=V_{2}=V$ 。 证明：化简销售商的期望利润 $\Pi\left(q, p_{1}\right)=\left(p_{1}-p_{2}\right) \int_{0}^{q} \bar{F}\left(\frac{x}{\bar{G}\left(\theta_{h}^{*}\right)}\right) d x-\left(c-p_{2}\right) q$, 对 $q$ 求偏导等于 0 , $q^{*}=\bar{G}\left(\theta_{h}^{*}\right) \bar{F}^{-1}\left(\frac{c-p_{2}}{p_{1}-p_{2}}\right)=\bar{G}\left(\theta_{h}^{*}\right) q_{0}^{*}$, 把 $q^{*}$ 代入 $\left(q, P_{1}\right)$, 可得 $\left(q^{*}, P_{1}\right)=\bar{G}\left(\theta_{h}^{*}\right) \Pi_{0}$ 。证毕。

比较 $q^{*}$ 及 $q_{0}^{*}$ 可知, 当客户对购买决策及购买时机有权衡行为时, 销售商的最优总体库存量会降低, 即 $q^{*}<q_{0}^{*}$ 。这是因为在双方理性博弯下, 销售商通过降低产品整体可得量来引导客户增加购买欲望。这与运 作实践中某些商家采用的限量发行、珍藏版策略相吻合。 $\mathrm{su}$ 和 Liu 等的研究中也得到了类似的产品配给 方面的结论。

结论 2: 当打折价 $P_{2}$ 固定时, 销售商存在一个最优旺季销售价

使其收益 $\Pi\left(q^{*}, p_{1}^{*}\right)$ 最大。

$$
p_{1}^{*}=\arg _{p_{1}} \max \left[\left(p_{1}-p_{2}\right) E\left(\bar{G}\left(\theta_{h}^{*}\right) x \wedge q\right)\right]
$$

证明：为了证明简洁，假设 $\mathrm{g}(\theta)$ 为 $[0,1]$, 均匀分布，则有

由销售商期望收益 $\Pi\left(q^{*}, p_{1}\right)$ 的一阶条件可得

$$
\bar{G}\left(\theta_{h}^{*}\right)=1-\frac{p_{1}-F\left(q^{*}\right) p_{2}}{\bar{F}\left(q^{*}\right) V}
$$

$$
\frac{\delta \prod\left(q^{*}, p_{1}\right)}{\delta p_{1}}=\int_{0}^{q_{1}} \bar{F}\left(\frac{x}{\bar{G}\left(\theta_{h}^{*}\right)}\right) d x-\left(p_{1}-p_{2}\right) \bar{F}\left(\frac{q^{*}}{\bar{F}\left(\theta_{h}^{*}\right)}\right) \frac{q^{*}}{\overline{F^{2}}\left(\theta_{h}^{*}\right)} \frac{1}{\bar{F}\left(q^{*}\right) V}
$$

上式第 1 项随 $P_{1}$ 递增, 第 2 项随 $p_{1}$ 递减, 因此 $\Pi\left(p_{1}\right)$ 一阶条件有唯一解, 证毕。

基于此, 接下来本文将在消费者对产品估值不统一的前提下, 讨论数量限制策略对客户策略行为的应 对。

\section{2 数量限制承诺}

当销售商采用数量限制承诺时, 即销售商承诺“此产品限量供应”, 人为制造其稀有性, 从而一方面维持 品牌形象, 另一方面也促使更多观望的消费者即时购买。比如著名的快速时尚服装品牌 ZARA 就对产品 线进行限制生产, 从而保证其在旺季销售一空, 这种策略也经常应用于高端的时装品牌。此时, 消费者 形成了产品数量有限的预期, 对产品的数量有比较确切的信息, 因此等待观望的可能性降低, 其能承受 的价格是产品数量的函数

本文用下标 $q$ 表示数量限制承诺下的变量。

$$
p_{q}(q)=\theta_{q} V-\left(\theta_{q} V-p_{2}\right) F(q)
$$

命题 3: 当销售商采用产品数量限制承诺时, 存在 1 个 $\theta_{q}^{*}$, 当消费者对产品的评价 $\theta$ 满足 $0>\theta_{q}^{*}$ 时, 则只 能在全价时购买。若消费者的评价 $0<\theta_{q}^{*}$ 时, 将放弃购买, 且有 $\theta_{q}^{*}=\frac{p_{q}-F(q) p_{2}}{\bar{F}(q) V}$ 。

此种情形下, 消费者的市场构成为: 部分评价高的消费者 $\bar{G}\left(\theta_{q}^{*}\right) x$ 并全价购买, 部分评价很低的消费者 $G\left(\theta_{q}^{*}\right) x$ 无法承受销售价格, 因此放弃购买。

对比有无数量限制承诺下的消费者组成, 可参见图 2 所示的消费者购买决策时序对比。 


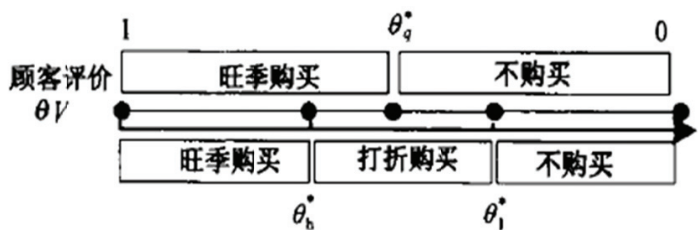

图 2. 消费者购买决策时序对比

观察 $\theta_{h}^{*} 、 \theta_{q}^{*} 、 \theta_{1}^{*}$ 之间的关系可知, 若销售商设定的 $p_{p} \leqslant p_{2}$, 则促使所有在旺季和打折购买的消费者 都在旺季购买, 但由于价格过低, 有可能损失收益; 若设定 $p_{p}=P_{1}$, 则销售商放弃了原打折购买的那部 分消费者, 而只抓取旺季购买的消费者群体; 若设定 $P_{2}<p_{p}<P_{1}$, 则取得原旺季和打折购买的总体消费 者的一部分，并促使其全部一次购买。

结论 3: 当销售商采用数量承诺, 且达到均衡时, 销售商采用以下的 $\left\{q_{q}^{*}, p_{q}^{*}\right\}$ 决策, 将最大化旺季销售收 益。 $P_{q}^{*}=\arg _{p q} \max \left[P_{q} E\left(\bar{G}\left(\theta^{*}\right) x \Lambda q\right)\right], q_{q}^{*}=\bar{G}\left(\theta^{*}\right) q_{0 q}^{*}$ 且 $\Pi\left(q_{q}^{*}, p_{q}\right)=\bar{G}\left(\theta^{*}\right) \Pi_{0 q}$

证明: 销售商的期望收益为 $\Pi\left(q_{q}, p_{q}\right)=P_{q} E\left(\bar{G}\left(\theta^{*}\right) x \Lambda q\right)-c q_{q}$, 经典报童模型下无打折时期望利润 $\Pi_{0 q}=P_{q} E(x \Lambda q)-c q_{0 q}$, 最优订货量 $q_{0 q}^{*}=\bar{F}^{-1}\left(\frac{c}{p_{q}}\right)$, 因此有 $q_{q}^{*}=\bar{G}\left(\theta^{*}\right) q_{0 q}^{*}$, 且 $\Pi\left(q_{q}^{*}, p_{q}\right)=\bar{G}\left(\theta^{*}\right) \Pi_{0 q}$ $P_{q}^{*}=\arg _{p q} \max \left[P_{q} E\left(\bar{G}\left(\theta^{*}\right) x \Lambda q\right)\right]$, 存在性证明类似, 此处略。证毕。

\section{5 结论}

将策略型消费者的理性购买行为引入到时尚服装供应链的运作策略分析中, 当消费者具有观望和等待的 理性行为后, 在满足消费者需求的前提下, 供应链的最优库存量有所降低; 为了应对消费者这种行为对 供应链绩效影响, 销售商采用了一些策略。本文在前人研究基础上, 放松了消费者对产品估值统一的假 设, 讨论了客户评价不一致下, 策略型客户行为对供应链的影响; 并进一步分析了数量限制策略的作用, 当销售商采用限量发行、珍藏版措施时, 客户对可得的产品数有比较准确的预期, 因此可能放弃等待, 即时购买。同时, 通过分析发现, 当销售商采取数量限制承诺, 并将产品在旺季的销售价格设定为 $P_{2}<p_{q}$ $<P_{1}$ 时, 能够实现收益最大化, 且有 $p_{q}^{*}=\arg _{p_{q}} \max \left[p_{q} E\left(\bar{G}\left(\theta^{*}\right) x \wedge q\right)\right]$ 。

本文探索把策略型消费者的理性行为引入到时尚服装供应链的分析中, 主要针对完全理性行为下的消 费者及销售商, 但在供应链决策的现实应用中, 供应链成员的有限理性行为更贴近实际, 也更值得研究。 同时, 本文重点研究单一阶段的决策过程, 如何将学习过程、信息更新等机制引人到双方的理性博亦模 式中, 也是一个值得研究的方向, 其可以更准确地描述供应链决策行为, 从而得出更为有益的结论。

致谢. 本文得到 2018 年湖北省社科基金一般项目（后期资助项目）(2018044)、武汉纺织大学 2018 年科 技创新计划立项（国家级项目培育计划-社科类）的资助，是其阶段性成果。

\section{参考文献}

1. 路应金,王静.消费者参与零售商共创环境下的供应链决策模式研究[J].物流工程与管理,2017,39(06):91-96

2. 曲佳莉, 胡本勇. 供应和需求不确定下考虑收益共享合约的供应链决策优化与协调研究 [J]. 电子科技大学学报(社科 版),2017,19(04):57-61+68

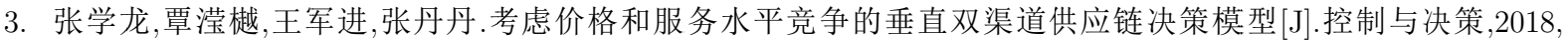
33(04):687-697

4. 李玮珩.零售商引入自有品牌对供应链决策的影响[J].合作经济与科技,2017(22):80-87

5. 苪鸿峰. 产出不确定环境下供需不平衡的供应链决策研究 [D].浙江工业大学, 2017

6. 张正林,凌静.基于消费者决策过程的电商定价策略研究[J].价格理论与实践,2014(11):117-119

7. 刘甜甜.电子智能产品营销策略之消费者决策心理研究[J].经贸实践,2018(15):200

8. 陈静. 基于顾客策略行为的替代品跨期定价研究 [D].西南交通大学, 2017

9. 李艳冰,汪传旭.基于成本利润率的两级供应链优化与协调[J].管理工程学报,2018,32(01):100-106 
10. 陈欢. 考虑制造质量的定制化供应链决策研究 [D].湖南大学, 2018

11. 王玉燕,于兆青. 考虑网络平台服务、消费者需求差异的混合供应链决策 $[\mathrm{J}]$. 系统工程理论与实践,2018,38(06): $1465-1478$

12. 张忠向,何军,吕毅.基于物流外包的零售企业供应链决策研究 [J].商业经济研究,2018(17):116-120 